\title{
On the Smith normal form of skew E-W matrices
}

José Andrés Armario

Departamento de Matemática Aplicada I, Universidad de Sevilla, Sevilla, Spain

\section{ABSTRACT}

Let $t$ be a positive integer. An E-W matrix is a square $(-1,1)$-matrix of order $4 t+2$ satisfying that the absolute value of its determinant attains Ehlich-Wojtas' bound. We show that the Smith normal form of every skew E-W matrix follows this pattern

$$
\operatorname{diag}[1, \underbrace{2, \ldots, 2}_{2 t}, m_{2 t+2}, m_{2 t+3}, \ldots, m_{4 t+2}]
$$

where $m_{2 t+3}>2$ and the product $m_{1} \cdots m_{k}$ divides $2^{2\lfloor k / 2\rfloor} t^{\lfloor k / 2\rfloor}$, for $1 \leq k \leq 4 t$.

\section{KEYWORDS}

E-W matrices; tournaments; Smith normal form

\section{AMS SUBJECT} CLASSIFICATIONS 05B20; 05C20; 15A23; 15A15

\section{Introduction}

Here and throughout this paper, for convenience, when we say determinant of a matrix we mean the absolute value of the determinant. Let $M$ be a D-optimal design of order $n$, that is, an $n$ by $n(1,-1)$-matrix having maximal determinant. Hadamard gave the upper bound $n^{n / 2}$ for $\operatorname{det} M$. This bound can be attained only if $n$ is a multiple of 4 . A D-optimal design that attains it is called a Hadamard matrix, and it is an outstanding conjecture that one exists for any multiple of 4 . For other orders more stringent bounds have been established. For $n \equiv 2 \bmod 4$, Ehlich [1] and independently Wojtas [2] proved that

$$
\operatorname{det} M \leq(2 n-2)(n-2)^{\frac{1}{2} n-1} .
$$

This bound can be attained only if $2 n-2$ is the sum of two squares. A D-optimal design that attains it is called an $E-W$ matrix. It has been conjectured that E-W matrices of order $n$ exist when $2 n-2=\alpha^{2}+\beta^{2}$ for some positive integers $\alpha$ and $\beta$. The construction and classification of Hadamard matrices and other D-optimal designs have become an important research field (see [3-6]).

Two $(-1,1)$-matrices are Hadamard equivalent provided one can be obtained from the other by a sequence of the following operations: row permutation, row negation and the corresponding column operations. The lack of a clear notion of a canonical form under this equivalence might explains the difficulty of classifying $(-1,1)$-matrices. For instance, the question of classifying Hadamard matrices is only solved for orders less than 36.[5] More generally, two integral matrices are integrally equivalent provided one can be 
obtained from the other by a sequence of the following operations: row permutation, row negation, addition of an integer multiple of one row to another and the corresponding column operations.

Obviously, Hadamard equivalence implies integral equivalence but the converse is false. On the other hand, integral equivalence has associated a clear notion of a canonical form but Hadamard equivalence does not.

Let $A$ be an integral matrix of order $n$ and $\operatorname{rank} r$. Then $A$ is integrally equivalent to a diagonal matrix

$$
\operatorname{diag}\left[a_{1}, a_{2}, \ldots, a_{r}, 0, \ldots, 0\right] \text {, }
$$

known as the Smith normal form of $A$, in which $a_{i}$ divides $a_{i+1}$ for $i=1, \ldots, r-1$. The diagonal elements $a_{1}, \ldots, a_{r}, 0, \ldots, 0$ are the invariant factors of $A$. They are uniquely determined up to sign.

One way to construct E-W matrices is to find two commuting $(-1,1)$-matrices $A$ and $B$ of order $2 t+1$ such that $A A^{T}+B B^{T}=4 t I+2 J$. Under this assumption the matrix

$$
M=\left(\begin{array}{cc}
A & B \\
-B^{T} & A^{T}
\end{array}\right)
$$

is an E-W matrix. In this situation, a bound for the minimum number of $2^{\prime} s$ which can appear in the Smith normal from of such matrices is provided in the following result.

Theorem 1.1: [7, Theorem 4] Let $M$ be an E-W-matrix in the form of (1). Then the Smith normal form of $M$ has one element 1 and at least $\left\lfloor\log _{2}(4 t+1)\right\rfloor+1$ elements equal to 2.

Remark 1: We cannot claim that every E-W matrix is equal (equivalent) to a matrix in the form of (1) (see [8]).

A $(-1,1)$-matrix $M$ of order $n$ is said to be of skew type if $M+M^{T}=2 I$, where $I$ denotes the identity matrix and $M^{T}$ the transpose of $M$. A Hadamard matrix $H$ of skew type is called a skew Hadamard matrix. Whenever a skew $(-1,1)$-matrix is mentioned in this paper, we mean a $(-1,1)$-matrix of skew type.

It was conjectured that skew Hadamard matrices exist for any order multiple of 4 . However, it was proved [9] that skew E-W matrices may only exist when $2 n-3=\alpha^{2}$ for some integer $\alpha$ (i.e. $\beta=1$ ), a condition which is believed to be sufficient. In [9] examples of skew E-W matrices for small orders have been provided.

The Smith normal form of every skew Hadamard matrix was computed in [10]. Analogously, we investigate the Smith normal form of every skew E-W matrices. Now the situation is more complicated and we have only obtained a partial result. We now state our main result.

Theorem 1.2: A skew E-W matrix of order $4 t+2$ has Smith normal form

$$
\operatorname{diag}[1, \underbrace{2, \ldots, 2}_{2 t}, m_{2 t+2}, m_{2 t+3}, \ldots, m_{4 t+2}] \text {, }
$$

where $m_{2 t+3}>2$.

Additionally, we will prove in the last section that the product $m_{1} \cdots m_{k}$ divides $2^{2\lfloor k / 2\rfloor} t^{\lfloor k / 2\rfloor}$, for $1 \leq k \leq 4 t$. Furthermore, if $t$ and $4 t+1$ are square free then $m_{2 t+2}=2$ and $4 t+1$ divides $m_{4 t+2}$. 
Notation. Throughout this paper upper case letters denote matrices, whose sizes are usually evident, in which case they are suppressed; otherwise $M_{r, s}$ denotes a matrix with $r$ rows and $s$ columns, and $M_{r}$ denotes $M_{r, r} . I$ is the identity matrix and $J$ is the all-ones matrix. $M^{T}$ denotes the transpose of $M$. The notation $\lfloor x\rfloor$ means the largest integer not greater than the real number $x$.

\section{Proof of Theorem 1.2}

The proof uses elementary linear algebra and falls naturally into a similar scheme to the proof of [10, Theorem 2]. Along this section we will introduce some definitions and results that we will need to conclude with the desired result.

An $E$ - $W$ tournament of order $4 t+1$ is a directed graph whose adjacency matrix $A$ satisfies (after permutations of row and columns)

$$
A+A^{T}=J-I, \quad A A^{T}=\left[\begin{array}{cc}
X & Y \\
Z & W
\end{array}\right] \quad \text { and } \quad A^{T} A=\left[\begin{array}{cc}
\tilde{X} & \tilde{Y} \\
\tilde{Z} & W
\end{array}\right]
$$

where

- $X=\left[\begin{array}{ll}X_{1} & X_{2} \\ X_{3} & X_{4}\end{array}\right]$ and $\tilde{X}=\left[\begin{array}{ll}X_{4} & X_{2} \\ X_{3} & X_{1}\end{array}\right]$ with

$$
X_{1}=t I_{t}+(t-1) J_{t}, \quad X_{4}=t I_{t}+(t+1) J_{t}, \quad X_{2}=X_{3}=(t-1) J_{t} .
$$

- $Y=\left[\begin{array}{r}(t-1) J_{t, 2 t+1} \\ t J_{t, 2 t+1}\end{array}\right]$ and $\tilde{Y}=\left[\begin{array}{r}t J_{t, 2 t+1} \\ (t-1) J_{t, 2 t+1}\end{array}\right]$.

- $Z=Y^{T}$ and $\tilde{Z}=\tilde{Y}^{T}$.

- $W=\left[\begin{array}{ll}W_{1} & W_{2} \\ W_{3} & W_{4}\end{array}\right]$ with

$$
W_{1}=t I_{\alpha}+t J_{\alpha}, \quad W_{2}=W_{3}^{T}=(t-1) J_{\alpha, \beta}, \quad W_{4}=t I_{\beta}+t J_{\beta},
$$

where $\alpha+\beta=2 t+1$ and $\alpha, \beta \geq 0$.

Example 2.1: The directed graph $T=(V, E)$ with adjacency matrix

$$
A=\left[\begin{array}{lllll}
0 & 1 & 0 & 0 & 0 \\
0 & 0 & 1 & 1 & 1 \\
1 & 0 & 0 & 1 & 0 \\
1 & 0 & 0 & 0 & 1 \\
1 & 0 & 1 & 0 & 0
\end{array}\right]
$$

is an E-W tournament of order 5.

The relationship between E-W tournaments and skew EW-matrices has been analysed in [11] and summarized in the following lemma.

Lemma 2.2: Given $M$ a skew $E-W$ matrix of order $4 t+2$. Then, 
- $M$ is Hadamard equivalent to a matrix of the form

$$
\left[\begin{array}{r|ccc}
1 & 1 & \cdots & 1 \\
\hline-1 & & & \\
\vdots & A^{T}-A+I \\
-1 & & &
\end{array}\right] .
$$

where $A$ is the adjacency matrix of an $E-W$ tournament with order $4 t+1$.

- $M$ is integrally equivalent to the direct sum $[1] \oplus 2\left(A^{T}+I\right)$.

Proof: The first step consists of multiplying suitable rows and columns of $M$ by -1 in such a way that the first row becomes the all-ones vector and the skew character does not change. After that, we construct a $(0,1)$-matrix $A$ of order $4 t+1$ as the submatrix of $\frac{1}{2}(J-M)$ obtained by deleting the first row and column. Finally, using block multiplication and the identity $M+M^{T}=2 I$ is showed in [11, Lemma 2.2] that $A$ satisfies (3).

Now, adding row 1 to all other rows, and then subtracting column 1 from all other columns in (4), it follows that $M$ is integrally equivalent to the direct sum [1] $\oplus 2\left(A^{T}+I\right)$.

In the following result, some immediate consequences of Lemma 2.2 are listed.

Corollary 2.3: Assume the same hypothesis and notation as in Lemma 2.2. We have:

- $\operatorname{det}\left(A^{T}+I\right)=t^{2 t}(4 t+1)$.

- The invariant factors $b_{1}, \ldots, b_{4 m+1}$ of $A^{T}+I$ and $m_{2}, \ldots, m_{4 t+1}$ of $M$ (where $M$ is of the form (4)) are related by $m_{i}=2 b_{i-1}$ for $i=2, \ldots, 4 t+2$.

- $m_{1}=1$.

The $p$-rank of an integer matrix $A$ is the rank of $A$ over a field of characteristic $p$ and is denoted by $\operatorname{rank}_{p}(A)$. The $p$-rank is considered a standard tool for studying the Smith normal form of $A$ since the $p$-rank of $A$ is related to the invariant factors $a_{1}, \ldots, a_{r}$ in a simple manner:

$$
\operatorname{rank}_{p}(A)=\max \left\{i: p \text { does not divide } a_{i}\right\} .
$$

In the following result, we investigate the $p$-rank of $A+I$ where $A$ is the adjacency matrix of an E-W tournament.

Lemma 2.4: Let $A$ be the adjacency matrix of an $E-W$ tournament of order $4 t+1$ and let $p$ be a prime divisor of $t$. Then $\operatorname{rank}_{p}(A+I)$ is equal to either $2 t$ or $2 t+1$.

Proof: Since $(A+I)(A+I)^{T}=A A^{T}+J$, from (3) by a simple observation it follows that $\operatorname{rank}_{p}\left((A+I)(A+I)^{T}\right)=2$.

$$
\operatorname{nullity}_{p}(A+I)=4 t+1-\operatorname{rank}_{p}(A+I) \geq \operatorname{rank}_{p}(A+I)-2 .
$$

Thus $\operatorname{rank}_{p}(A+I) \leq 2 t+1$.

On the other hand, using properties of the ranks and (3) it follows that

$$
\begin{aligned}
4 t & =\operatorname{rank}_{p}(J+I)=\operatorname{rank}_{p}\left((A+I)+(A+I)^{T}\right) \\
& \leq \operatorname{rank}_{p}(A+I)+\operatorname{rank}_{p}(A+I)^{T}=2 \cdot \operatorname{rank}_{p}(A+I),
\end{aligned}
$$

which implies that $\operatorname{rank}_{p}(A+I) \geq 2 t$. Therefore $\operatorname{rank}_{p}(A+I)$ is either $2 t$ or $2 t+1$. 
Corollary 2.5: Assume the same hypothesis and notation as in Lemma 2.4. If $p$ divides $b_{k}$ (the $k$-th invariant factor of $A+I$ ) then $k>2 t$.

Proof: Combining that $\operatorname{rank}_{p}(A+I)<k$ by (5) and Lemma 2.4.

Finally, assuming that $p$ is a prime divisor of $b_{2 t}$ and taking into account that $b_{1} \cdots b_{4 t+1}=$ $\operatorname{det}\left(A^{T}+I\right)$. Then either $p$ is a divisor of $t$ or else $p$ is a divisor of $4 t+1$ and $p \geq 3$. We rule out the first case by Corollary 2.5. In the second case, $p$ is a divisor of $b_{i}$ for $i=2 t+1, \ldots, 4 t+1$, so $p^{2 t+1}$ should divide $4 t+1$, but this is contrary to $p^{2 t+1}>4 t+1$ for all $t \geq 1$. Therefore $b_{2 t}=1$ and $b_{2 t+2}>1$, for then $m_{2}=m_{3}=\ldots=m_{2 t+1}=2$ and $m_{2 t+3}>2$. Let us point out that if $t$ is square free then $m_{2 t+2}=2$. This concludes the proof.

\section{Conclusions}

In this paper we have proved that if $p$ divides $t$, then the $p$-rank of $A+I$ is either $2 t$ or $2 t+1$ where $A$ is the adjacency matrix of an E-W tournament of order $4 t+1$. There is a one-to-one correspondence between this kind of tournaments and skew E-W matrices. [11] We can always assume without loss of generality that every skew E-W matrix is of the form (4). Examples of skew E-W matrices for small orders have been provided in [9].

As a consequence, we have showed that the invariant factors $\left(m_{1}, \ldots, m_{4 t+2}\right)$ of every skew E-W matrix satisfy that

$$
m_{1}=1, m_{2}=\cdots=m_{2 t+1}=2 \text { and } m_{2 t+3}>2 .
$$

Taking into account the spectrum of $A$ and of $M$, we obtain the following property about the products of invariant factors.

Proposition 3.1: $\quad m_{1} \cdots m_{k}$ divides $2^{2\lfloor k / 2\rfloor} t^{\lfloor k / 2\rfloor}$, for $1 \leq k \leq 4 t$.

Proof: First, we consider that $k$ is odd. The proof is based on the following facts:

- In $[11$, p. 10] we conjectured that the existence of skew E-W matrices is equivalent to the existence of tournament matrices with a certain spectrum. Concretely, $\frac{-1 \pm \sqrt{1-4 t}}{2}$ are eigenvalues each with algebraic multiplicity $2 t-1$. Recently, this conjecture has been proven in [12]. Thus, $\frac{1 \pm \sqrt{1-4 t}}{2}$ are eigenvalues of $A^{T}+I$ each with algebraic multiplicity $2 t-1$.

- $b_{1} b_{2} \cdots b_{k}$ divides the product of any $k$ eigenvalues of $A^{T}+I$ (repetitions allowed), in the sense that the quotient is an algebraic integer, for $1 \leq k \leq 4 t+1$ (see [13]).

- The identity $\left(\frac{1+\sqrt{1-4 t}}{2}\right)^{l} \cdot\left(\frac{1-\sqrt{1-4 t}}{2}\right)^{l}=t^{l}$, for any positive integer $l$.

- The only algebraic integers which are found in the set of rational numbers are the integers.

Hence, $b_{1} \cdots b_{2 l}$ divides $t^{l}$ for $1 \leq l \leq 2 t-1$. Now, the desired result follows immediately where $k=2 l+1$.

The result for $k$ even follows mutatis mutandis since $1 \pm \sqrt{1-4 t}$ are eigenvalues of $M$ each with algebraic multiplicity $2 t$ (see [12, Lemma 3.3]).

Finally, after looking over small orders, we conjecture that every skew E-W matrix of order $4 t+2$ has Smith normal form 


$$
\operatorname{diag}[1, \underbrace{2, \ldots, 2}_{2 t+1}, \underbrace{2 t, \ldots, 2 t}_{2 t-1}, 2 t(4 t+1)] .
$$

Obviously, this conjecture holds when $t$ is prime number and $4 t+1$ is square free. The first example of skew E-W matrix satisfying this hypothesis is provided for $t=3$. This and Proposition 3.1 weigh heavily in favor of the conjecture.

Consequently, if this conjecture would be proven, we will not be able to distinguish among skew E-W matrices of the same order $4 t+2$ using the Smith normal form. On the other hand, if an E-W matrix $M$ has a Smith normal form different to (6), then $M$ cannot be equivalent to a skew E-W matrix.

\section{Acknowledgements}

The author would like to thank Kristeen Cheng for her reading of this manuscript.

\section{Disclosure statement}

No potential conflict of interest was reported by the author.

\section{Funding}

This work has been partially supported by the research project FQM-016 from JJAA (Spain).

\section{References}

[1] Ehlich H. Determiantenabschätzungen für binäre Matrizen. Math. Z. 1964;83:123-132.

[2] Wojtas W. On Hadamard's inequallity for the determinants of order non-divisible by 4 . Colloq. Math. 1964;12:73-83.

[3] Álvarez V, Armario JA, Frau MD, et al. Embedding cocyclic D-optimal designs in cocyclic Hadamard matrices. Electron. J. Linear Algebra. 2012;24:66-82.

[4] Kharaghani K, Orrick W. D-optimal matrices. Colbourn C, Dinitz J, editors. Handbook of combinatorial designs. 2nd ed. Discrete Matematics and its applications. Boca Raton (FL): Chapman \& Hall/CRC; 2007. p. 296-298.

[5] Kharaghani H, Tayfeh-Rezaie B. On the classification of Hadamard matrices of order 32. J. Combin. Des. 2010;18:328-336.

[6] Orrick W, Solomon B. The Hadamard maximal determinant problem. Available from: http:// www.indiana.edu/ maxdet/.

[7] Koukouvinos C, Mitrouli M, Seberry J. On the Smith normal form of D-optimal designs. Linear Algebra Appl. 1996;247:277-295.

[8] Cohn JHE. On the determinants with elements \pm 1 II. Bull. London Math. Soc. 1989;21:36-42.

[9] Armario JA, Frau MD. On skew E-W matrices. J. Combin. Des. 2016 [published online]. doi:10.1002/jcd.21519

[10] Michael TS, Wallis WD. Skew-Hadamard matrices and the Smith normal form. Designs, codes Cryptography. 1998;13:173-176.

[11] Armario JA. On $(-1,1)$-matrices of skew type with the maximal determinant and tournaments. In Colbourn CJ editor. Algebraic desing theory with Hadamard atrices. Springer Proceedings of mathematics and statistics. 2015. Vol. 133, p. 1-11.

[12] Greaves G, Suda S. Symmetric and skew-symmetric $\{0, \pm 1\}$-matrices with large determinants. Available from: http://arxiv.org/abs/1601.02769.

[13] Rushanan JJ. Eigenvalues and the Smith normal form. Linear Algebra Appl. 1995;216:177-184. 